\title{
Disability Mainstreaming: Practices and Challenges among Selected Governmental Organizations of Bahir Dar City Administration
}

\author{
Aytenew Adamu \\ Debre Tabor University, Ethiopia
}

\begin{abstract}
Disability mainstreaming program is a newfangled strategy devised to enhance the rights of PWDs and create disability-inclusive development. As the program is new, in Ethiopia researches on its practice are limited. Therefore, to increase body of knowledge towards the program, this case study research conducted on the practice of disability mainstreaming in account of challenges for implementation among three selected government organizations of Bahir Dar city. Interview and FGDs were used to collect data. The collected data were analyzed using thematic analysis technique. As the findings revealed, there are visible and encouraging trends regarding the practice of disability mainstreaming. Study sectors strive for disability-inclusive development through devising appropriate programs, building human capacity, creating linkages, appointing disability focal person and developing manuals and checklists. Although, there are the aforementioned initiations in practicing disability mainstreaming, some deterring factors put a shadow on these efforts. Factors like, absence of ministry for disability issues, dearth of involvement of PWDs and DPOs, absence of budgeting and institutional and team loafing restrained the practice of mainstreaming. Besides, in the study sectors the practice of mainstreaming affected by lack of awareness, slack follow-up and nominal power of responsible organizations, poor documentation and reporting and hopelessness and sentimentality. The study recommends awareness creation activities have to be done to change the pejorative attitudes of service providers and passive character of service users. Collaborative efforts should be implemented by different stakeholders to realize the effectiveness of the program. Besides, newfangled and all-inclusive trainings should be given for managers, disability focal persons, staffs and service users to facilitate opportunities for disability equality.
\end{abstract}

Keywords: Disability Mainstreaming, Disability, Peoples with Disabilities, Inclusive Development, Bahir Dar DOI: $10.7176 / \mathrm{JCSD} / 61-04$

Publication date:September $30^{\text {th }} 2020$

\section{INTRODUCTION}

Disability mainstreaming is the current international approach to advancing the rights of Peoples with Disabilities (PWDs) and creating an inclusive environment in every sphere of the development. It is a strategy for making disabled people's concerns and experiences an integral dimension of the design, implementation, monitoring and evaluation of policies and programs in all political, economic and societal spheres so that disabled people benefit equally and inequality is not perpetuated (Albert, Dube \& Hansen , 2005).

The 2006 international declaration of United Nations convention of rights with disabilities (UNCRPD) was the first convention which brings the concept of Disability mainstreaming into agenda and came into force on 3 may 2008. This convention marked a paradigm shift and a change of attitude towards PWDs with a view to fixing society, not people. Moreover, the convention declares disability is an issue to be considered in all programs rather than stand-alone as thematic issue, and requires every nation to take measures that ensure full and equal participation of PWDs in society. Specifically article 32 of the Convention of Rights of Peoples with Disability (CRPD) states all development corporations should be all inclusive (UN, 2011).

Disability mainstreaming as a strategy of incorporating disable people in all development plans and programs become widely accepted by governments throughout the world for achieving disability equality (UN, 2011). With $15 \%$ of the world's total population and $20 \%$ of the world's poor made up of PWDs, mainstreaming is an important policy agenda, not only from a human rights point of view, but also from a broader economic development perspective (The Economist Intelligence Unit,2014). Ethiopia has become part of this global community for safeguarding the rights of disable people. In supporting the rights of PWDs, the government of Ethiopia adopted and implemented different laws, policies, proclamations and standards. For instance, the 1995 constitution of FDRE clearly stipulates the rights of PWDs (FDRE constitution, 1995).

Besides, in order to create conducive environment in which PWDs enjoy their rights and access equal opportunities with their non-disabled peers' proclamations also ratified. Proclamations like Labor proclamation No. 494/2006, The FDRE civil servant proclamation, No. 515/2007, Proclamation concerning the rights to employment for PWDs, No. 568/2008, Building proclamation 624/2009, and proclamation No. 676/2010 of United Nations conventions of rights of PWDs ratification by Ethiopia were announced (ILO, 2013). Furthermore, the government provides special considerations on its development plans through providing special concerns in its 
growth and transformation plan (GTP) (2010-2015) and adapts the national plan of action of PWDs (2012-2021) which point towards creating an inclusive environment (Ministry of Finance and Economic Development [MoFED], 2010). The aforementioned laws, polices, proclamations and plans be evidence for the entire efforts that has been made by the Ethiopian government to make disabled people contributory for achieving the development goals of the country.

Almost all of these documents embark up on the need of non-discriminatory treatments, full and effective participations, equal opportunities and respect for the rights and evolving capacities of PWDs (ILO, 2013). However, in Ethiopia the practical implementations of these inspirational objectives in the real ground remains in question. In this regard, Ministry of Labor and Social Affairs ([MoLSA], 2009) in its national employment policy and strategy paper describes the indicator that shows the discriminations that PWDs faces both in the formal and informal institutions. The policy stated that because of the special physical conditions and needs, PWDs are facing peculiar challenges that expose them to exclusion and poverty.

Kassahun (2013) argued that the issue of putting these into practice to ensure the rights of PWDs and create equal access and inclusive environment for PWDs is still in its infancy. Even with the presence of the stated laws, policies and proclamations their needs are ignored and considered as needles, segregated in institutions, become voiceless in different spheres, denied from political, economic and educational involvements and excluded from the community. Mindahun (2006) also confirmed that the extent to which changes in legislation and documentation reflect change in practice is more debatable. According to his research, in the presence of the proclamation No. 568/2000, which declares the rights to employment of PWDs; the blind and physically impaired groups are still discriminated. Some had been discriminated on training, others on promotion and even some others in their workplaces after they employed. This presents that merely declaring of laws and policies towards the protection of the right of PWDs to employment is not a guarantee to safeguard their rights.

Thus, to overcome this problem the government adapted the national plan of action of PWDs (2012-2021) to include disability in all our work through mainstreaming disability. It is in the assumption that mainstreaming starts with us. We can all be role models of more equitable and inclusive relationships between disabled and nondisabled people (ILO, 2013). These inconsistencies of formulated laws and their implementations to the ground motivated me to identify disability-mainstreaming program as a research area for my thesis. Thus, this study explored the practice of disability mainstreaming among Bahir Dar city education office, Bahir Dar city health office and Bahir Dar city technical, vocational, education, training and small enterprise office located in Bahir Dar city administration. The following are the main research questions of this study:

- How disability-mainstreaming program is implementing in the selected study areas?

- What are the challenges that restrain the practice of disability mainstreaming in the selected organizations?

The objective this study was to explore the practice of disability mainstreaming in the selected three governmental organizations of Bahir Dar city administration. Specifically this study focused on describing the practice of disability mainstreaming in the selected study areas. The study also aimed at exploring the challenges for mainstreaming disability among selected government organizations. The targets of the study were Bahir Dar city education office, Bahir Dar city health office and Bahir Dar city technical, vocational, education, training and small enterprise office located in Bahir Dar city administration

\section{Conceptualizing Disability Mainstreaming}

One of the points frequently made by advocates for mainstreaming disability in development is that disability needs a treatment by both agencies and governments as a crosscutting issue. Disability mainstreaming is incorporating the issues of marginalized portion of the population into the design of all policies, measures, and the action to address the problem of PWDs is to be limited to those policies and measures that are specifically addressing their needs (The Economist Intelligence Unit, 2014).

The general goal of mainstreaming disability is to cement disability issues as a cross cutting concern to be considered in all development agendas and create a comprehensive environment which PWDs enjoy their rights and equal opportunities (United Nations Economic Commission for Africa \& Leonard Cheshire Disability, 2008). The United Nations CRPD also declares development cooperation's should create disability inclusive settings. Specifically article 32 of the convention states that:

International cooperation plays an important role in promoting and ensuring the realization of the Convention. Measures to ensure this could be to: "Ensuring that international cooperation, including international development programs, is inclusive of and accessible to persons with disabilities" (HANDICAP international, 2009, p.19).

In the same fashion, the document from BoLSA (2007) states the goal of mainstreaming as making governmental organizations, NGOs, civic organizations and private companies incorporate disability issues into their development policies, strategies and plans and strive for implementation. 


\section{Key Areas of Mainstreaming}

Jones and Webster (2006) developed a VSO program-mainstreaming model that helps as a framework for development organizations to mainstream disability in their organization and area of work. The model identifies five basic areas by which disability incorporated to attain disability equality in different programs and organizations. According to this model, effective disability mainstreaming takes place in organizational commitment, sensitization, workplace mainstreaming, program mainstreaming and policy.

United Nations Relief and Work Agency (2013), also identifies the areas which disability mainstreamed to promote inclusion and tackle the barriers that exclude persons with disabilities from the equal enjoyment of their human rights. To this agency, all fields should consider disability as cross cutting issue and incorporate in all areas of their practices. In order to achieve the rights of PWDs to enjoy their rights and opportunities equally with their normal peer's disability mainstreamed within programs, project design, policies, protocols and procedures as well as in staff training. For Swedish International Development Agency (SIDA) (as cited in Miller \& Albert, 2005) mainstreaming has takes place at the general level of the agency itself in accordance of its culture, policies and practices, within the programs of the agency and within the outputs to improve disability equality in every aspects of the agency.

Theses indicates that, so as to achieve disability equality in all spheres of life everybody has to be participated in working towards disability and mainstreaming needs wider organizational support and management commitments in all programs, policies and strategies of development. Mainstreaming would not be the activities of selected individuals and organizations rather it has to be included in all aspects of every organization and practiced by all individuals.

\section{Challenges for practicing disability mainstreaming}

It is bitter facts that PWDs are in need of special attention and considerations, but they are still neglected and kept away from the involvement and fruits of development. As a result, still date, they are one of the most marginalized and impoverished groups within the society and are more likely to be uneducated, unemployed, face lack of access to healthcare, remain vulnerable to abuse and socially marginalized (CART, 2011). Disabled people, in all parts of the world, experience discrimination and are widely excluded from the social, economic and political life of the community. This exclusion is the basic cause of high rates of poverty among disabled people in the poorest countries. There is a close link between poverty and disability in the sense that poverty often causes disability and disability increases poverty (Dublin, 2004.). The relationship that exists between poverty and disability often considered as a vicious circle. Here, it argued that both disability and poverty are often manifestations of the same processes. If the commonalities are recognized then the need to build horizontal alliances becomes more apparent (Yeo, 2005).

The past and present neglect of PWDs differences in general development programs, policies and legislation had negative impacts on the process of disability mainstreaming. For instance, one such negative impact at the policy level is that the MDGs, which are the basis for the majority of development strategies, do not mention people with disabilities (HANDICAP, 2009).

The MDGs which puts poverty, exclusion, environmental problems, education, gender equality, child mortality, mental health, HIV/AIDS, malaria and other diseases and global partnership at the center of their considerations but disability has not been identified in these MDGs, which grounds for different protests from the advocators of disability equality(Light for the World, 2012). Even though the MDGs do not put a strategic plan to address disability issues, its link with the aforementioned MDGs makes implicitly significant to address the rights of PWDs. The assumption is that as disability influences the livelihoods of PWDs and leads to chronic poverty, working to eradicate poverty reduces vulnerability of disabled people. It is known that PWDs are less likely to attend their education, women`s with disabilities bear double burden, adults and children with disabilities are more vulnerable to accidents, PWDs are significantly vulnerable to become HIV-infected and excluded from the community. However, the MDGs do not specifically address the problem of disability; the problem is touched in all pillars (UN, 2006).

The link between disability and poverty has however been clearly identified in numerous research and reports. There is a strong bi-directional correlation between disability and poverty. According to the World Bank (WB) report (as cited in Bruijn, 2013) estimated that PWDs accounts 15\% of the total population of the world and from this portion of population $80 \%$ of PWDs are estimated to live on less than one Euro a day and only about $2 \%$ have access to basic services. Persons with disabilities are therefore at particular high risk of being poor, and being poor constitutes a specific risk for disability. These presents PWDs account for a significant part of the population and are more likely to live in poverty than their able-bodied peers.

The United Nations Development Program (UNDP) states that development can be inclusive and reduce poverty effectively only if it is inclusive for all (UNDP, 2014). A vast majority of PWDs live in rural areas where access to basic services is limited. In Ethiopia, 95 per cent of all PWDs estimated to live in poverty. Many depend on family support and begging for their livelihoods (ILO, 2013). From the above explanations, one can deduce 
that there is a strong bondage between disability and poverty. Eliminating poverty from the ground is ineffective without addressing the rights and needs of PWDs in different areas of development. Similarly, addressing the rights and needs of PWDs requires the allocation of adequate resources for disability issues. PWDs play an integral role in the development process of the community and the country at large if their issues taken into account.

A qualitative study conducted on the issue of mainstreaming by Mohapatra also finds that difficulty in accessibility, discrimination, lack of education and skills of mainstreaming, stakeholders views of disability as a charity and welfare issues and their lack of awareness on disability rights are major factors that challenge the process of disability mainstreaming in India. Weak implementation and other institutional changes have also turned the practice of disability mainstreaming into roadblocks that have halted change on disability issues (Mohapatra, 2012).

\section{Research Method \\ Description of the Study Area}

This study was conducted in Bahir Dar city. Bihar Dar city is the capital of the Amhara national regional state in the federal democratic republic of Ethiopia. Metropolitan Area of Bahir Dar found in the Bahir Dar Zuria Woreda. It is specifically located in the central part of Amhara National Regional State encircling the periphery of Lake Tana's southern tip. The metropolitan area of Bahir Dar stretches about $25 \mathrm{~km}$ radius from the center of Bahir Dar city proper. The city has a road distance of $567 \mathrm{Kms}$ from Addis Ababa (Tana forum, 2015).

According to the population and household census of 2007, the population of Bahir Dar is 220,344 /including rural kebeles/. When disaggregated by place of settlement, the rural population of Bahir Dar constituted 40,250 while the urban population is 180,094 . In terms of gender composition, the female population of Bahir Dar is slightly higher than the number of male population. Out of 180,094 urban populations, 93,005 are female and 87,089 are male. Annual population growth of the city is about $6.6 \%$. From this $2.6 \%$ is the birth rate and $2.8 \%$ is migration rate (Central Statistics Agency [CSA], 2007).

In the city, there are 1617 PWDs. From the total population of PWDs the magnitude of specific disabilities in the city is as follows: 502 persons with motor disorders, 178 persons with hand disability, 328 persons with visual impairment, 105 persons with hearing impairment, 189 persons with cognitive disability, 78 persons with speech and language impairments, 94 persons with multiple disabilities and 143 others. According to this survey, persons with motor disorders comprise the largest group followed by persons with visual and hearing impairments (BoLSA, 2015).

In the city, there are around 32 governmental organizations, which the government enacted the program of disability as their responsibilities under taken by these sector bureaus. In each of this organization, the issue of disability received a legal recognition to incorporate in the planning, design and implementation aspects of development. From these sector bureaus, only 23 sectors were practicing disability mainstreaming program to the ground (Alem, personal communication, February 4, 2016). From these government organizations, based on some criterions of inclusion this study bounded only to Bahir Dar city education office, Bahir Dar city health office and Bahir Dar city technical, vocational, education, training and small enterprise office located in Bahir Dar city administration.

\section{Methods and Materials}

In this study the researcher tries to explore the practice of disability mainstreaming and investigate the challenges for the practice of disability mainstreaming program. For this purpose, data were collected through semi structured interviews and focus group discussions. Semi-structured interviews were chosen due to their advantage over providing detail information about the case, their ability to produce focused data, and their potential to elicit information from few participants (Matthews and Ross 2010). Participants of the interview were selected purposively on the assumption that when office heads, deputy heads and focal persons working in their respective organizations provide profound and reliable data. Accordingly, interviewees were selected from Bahir Dar city education office, Bahir Dar city health office and Bahir Dar city technical, vocational, education, training and small enterprise office located in Bahir Dar city administration. For this reason, office heads, deputy heads and focal persons totally Eight individuals who coordinate disability mainstreaming in the target sectors of the study area were take part for this study and interviewed. Beside, two individuals, one from BoLSA and one from the federation of PWDs were also participated in this research as key informants. It is in the assumption that as BoLSA takes the responsibility of disability mainstreaming and coordinates other sectors for practice gives an in-depth data about the program as a result the researcher incorporates the manger of the organization as a key informant for the study. The manger of the federation was also participated in this research as a key informant since the practice of disability in general and disability mainstreaming in particular is their direct concern. To triangulate the data acquired through interview three focus group discussions (FGDs) also conducted. Each FGD group consists of six members. For FGDs, eighteen participants selected through employing convenience-sampling techniques. These discussants were planners and experts who are working in the respective organizations under 
scrutiny. Totally, for this research 26 participants ( 8 for interview and 18 for FGD) were involved. Thematic analysis was employed to analyze the collected data.

Regarding research ethics, participants were informed about the objective of the study and consent was obtained from each participant. Besides, their names are anonymous in the study to secure their confidentiality.

\section{Result and Discussion}

The results obtained from this study focuses on the practice of disability mainstreaming program and the challenges that hinder the implementation of disability mainstreaming in the study area.

\section{Practice of Disability Mainstreaming}

It is evident from the findings of this research that the study sectors devised various strategies of disability mainstreaming which are inclusive, effective, gainful and meaningful for PWDs in order to promote meaningful and effective inclusion of PWDs. For incorporating disability issues into all aspects of development, the study sectors designed appropriate programs, which address the interests of PWDs and build the capacity of human resources for quality service provision. To facilitate the process of disability mainstreaming program sectors also create linkages with various governmental, non-governmental and civic organizations for joint action. Delegating disability focal person and developing mainstreaming manuals and checklists, which are essential to create disability- inclusive environment are also the other practical strategies used by the selected study bureaus. Generally, the efforts done by the study sectors for practicing disability-mainstreaming program are falling under the following strategies of disability mainstreaming:

\section{Designing appropriate programs}

As PWDs are facing with multiple difficulties resulting from their impairments, study sectors devised different strategies to create an inclusive development. Participants of the research explained PWDs are included into development aspects of the country through developing two broad sets of initiatives. One set is through disabilityspecific activities that targeted directly for people with a disability; the other is through the mainstreaming of disability into broader activities. In this regard, an interview with disability focal person of education office has the following to say:

For PWDs, our sector is developing units for special need education program to address the problem of students with disabilities. Besides, for access and quality education the sector is developing a resource block center which helps in preparing teaching aid materials, giving non-curricular acuities such as for visually impaired children, mobility and orientation training, Braille reading and writing, daily living skills activities, pre Braille trainings etc. further students with disabilities deliver monthly and annual stipends for their financial requirements.

Participants explained there is an immense effort towards creating equal and accessible environment for PWDs. Students with disabilities are equipped with material assistances, financial supports and developing resource centers that uniquely address the needs of PWDs. The manager of health sector also explained the same thing differently. He confirmed special medical programs, which targeted specific impairments or causes of impairment developed in the health sector. He noticed, "A number of interventions to address blindness and its causes, including cataract and trachoma are frequently practiced". Provision of such special programs for PWDs narrows the gap, which existed between PWDs and their non-disabled peers. It also reduces the vicissitudes of PWDs, make services easily accessible for them and beneficiaries of the available services.

Beyond organizational implementations, special programs also practiced at the community level. Organizational systems created that make the community work collaboratively with the study sectors to implement special programs that designed to address the problems of PWDs. At the community level, PWDs received and recruited for special programs. A focal person in TVET office explained that:

In each Keble'-s there are committees working in collaboration with Community Care Coalition (CCCs) to opt for eligible disabled people to work. The Keble'-s recruited eligible disabled vacant and refer to our sector for employment. Then the TVET bureau organizes and facilitates market stocks and credits for the entitled PWDs.

The data from FGDs also confirmed the availability of inclusive and special programs designed to benefit PWDs. Nevertheless, the discussants of the research are afraid on the implementation of designed programs to the ground. Expressly, special programs, which uniquely devised for PWDs were not implemented beyond paper work. Because of inadequate resources and negligence of responsible bodies of PWDs, particularly those who are in need of special treatments resulted from their multiple impairments forced to equal treatment with non-disabled service beneficiaries.

\section{Capacity building}

According to the data obtained from interviews and FGDs study sectors made efforts to develop the capacity of 
human resources for facilitating the practice of mainstreaming to the ground. Some of the study participants indicated that, at the outset efforts made to increase the familiarity of the focal persons and staffs on disability mainstreaming through perspective building and developing a knowledge base. The manager of BoLSA reflected, "To inculcate managers and disability focal persons with the program relevant trainings and workshops on disability, which incorporates human rights principles provided. In-service trainings also provided to encourage quality service provision". The deputy manager of education office has also explained the effort made to improve the capacity of human resources in the education sector. To implement disability mainstreaming and create inclusive environment teachers at different schools provided with different trainings.

The data obtained from an interview with disability focal person, manager of federation of PWDs and discussants of the FGDs reflected different views from the above statements concerning the capacity buildings provided for focal persons and staffs of the selected sectors. Disability focal person of health office explained, "There were some homonym trainings provided at the end of each year by BoLSA for the managers and focal persons on disability mainstreaming in the study sector bureaus". For her the training conducted just for makeweight the annual reports for the higher authorities than equipping managers and focal persons with newfangled and pertinent knowledge and skills of mainstreaming. The manger of federation of PWDs also explained his dissatisfaction with the efforts made by the study sectors to build the capacity of service providers. He complained about the limited practice of capacity building both in type and in target. According to him, "Most of the sectors strived to build the capacity of service providers only through conducting workshops and trainings for managers and disability focal persons. But the program can be easily practiced if community mobilization activities are consolidated". He added, "The beneficiaries of the program specifically PWDs were not part of taking such capacity building trainings and workshops". This means service users are not clearly accustomed with the program of disability mainstreaming which designed for creating an inclusive environment for PWDs. Such awareness gaps of PWDs make them not to insist on their rights and responsibilities in the mainstreaming process. This is to mean although efforts in building the capacity of human resources were made, they were homonym in character and not encompassed service providers and service users for quality service interaction.

\section{Creating linkage}

As a strategy for practicing disability mainstreaming, the study sectors made an effort for creating linkages with other organizations through developing different projects and programs. Data obtained from participants depicted study sectors developed a connection with other governmental, non-governmental and civic organizations for joint action on disability. The linkage becomes a source of material assistance, financial and technical support and good experiences. In this regard, the deputy manager of TVET office stated, "Our sector were made linkages with organizations and cabinets, shared experiences, avoid repetitions and developed an organized system of documentation". The study sectors used their networks as a source of sharing experiences, which are helpful for directing the right track of practicing the program.

\section{Assigning disability focal person}

The study sectors also practiced disability-mainstreaming program through assigning focal person. The results portrayed that study sectors primarily assign disability focal person that considered as leading man for disability mainstreaming before practicing the program. In this regard, the manager of education office reported, "From the outset, our sector started mainstreaming disability through assigning one expert as focal person of disability mainstreaming". Designating disability focal person is a good beginning for practicing disability mainstreaming because this assigned person is a principal and resource center in each sector bureaus. The disability focal person coordinates other staff members and service users for joint action. The data obtained from an interview with a focal person of health office also affirmed the presence of assigned disability focal person in the health sector.

\section{Developing disability mainstreaming manuals and checklists}

Study sectors were developed mainstreaming manuals and checklists for facilitating the practice of the program. According to the study participants, study sectors developed or contextualize mainstreaming manuals and checklists in accordance with their vision and mission.

Regarding this, the manger of BoLSA described:

Our sector developed a clear and precise disability-mainstreaming manual that encompassed the responsibilities of 23 governmental sector bureaus of Bahir Dar city administration in dealing with disability mainstreaming and disability equality. This manual distributed for all of these organizations as a guideline of mainstreaming.

The disability focal person of TVET office has also the following to say:

In one hand, there is a disability-mainstreaming manual developed by Amhara regional state BoLSA that directed our sector with its responsibilities in mainstreaming disability into different programs, plans and strategies of development. On the other hand, our sector bureau developed their own disability 
mainstreaming manuals and checklists in accordance with the values, vision and mission of the organization that make brief the responsibility of staffs in mainstreaming disability.

The data obtained from the above interviews stressed on the existence of checklists and manuals developed by BoLSA, the responsible organ of disability mainstreaming program and by each sector bureaus of the study. This manuals and checklists are important instruments for putting disability mainstreaming into practice. The documents make brief the duties and responsibilities of each sector bureaus and practitioners. At the same time, they create sense of accountability on practitioners and make disability issues part of development practices.

The data acquired from discussants of FGDs shows a different perspective from the above interviews. They stressed that manuals and checklists might be at the hand of managers and disability focal persons of the sector bureaus but not disseminated for staffs of the sector offices. Because of such gaps in identifying their responsibilities, discussants are facing with difficulties in planning, implementing, monitoring and evaluation of disability mainstreaming. This shows that disability mainstreaming manuals and checklists remained at the hand of managers and focal persons. It is to mean that the manuals were not disseminated for staffs of the study sectors.

\section{Challenges for Disability Mainstreaming}

Multifaceted and interconnected factors deterred the practice of disability mainstreaming in the study sectors. The data indicated that the practice of disability mainstreaming is be swayed by institutional, structural, personal and inaction problems. It is evident from the data that the program of disability mainstreaming in the study sectors failed to have a responsible structure that runs the issue of disability at all levels. PWDs are not taking part in planning and decision making activities which in turn makes disability issues as a pity done by others and highly susceptible to budgetary restraints. The program of disability mainstreaming faced with awareness gaps from its practitioners and service receivers that deteriorated the practice of disability mainstreaming. Lack of awareness resulted institutions and teams escaped from taking disability responsibilities and designed poor documentation and reporting strategies. The program also challenged by lack of devised strategies, which clearly dictate accountability of staffs and sector bureaus. Further, disability-mainstreaming program is also hindered by the passive nature of service users to insist and resist for their rights and equal opportunities in the process of implementing the program.

\section{Absence of ministry for disability affairs}

Participants complained on the absence of central disability unit with policy responsibilities and mandate to oversee the overall disability issues in general and disability mainstreaming process in particular. Although there are disability focal persons in each sector bureaus of the study, there is no clear institutional structure that developed for coordinating focal persons for action, dealing disability issues and oversee the situation of PWDs and their programs. The focal person of TVET office noticed, "Disability issues are being dealt with an ad hoc basis because of absence of ministry which ensures the coordination of disability issues." He further explained, "Because of absence of administrative head on disability issues responsibilities' of policy formulation and review for PWDs; and regulations of the policy implementations are made arbitrarily. Ensuring mainstreaming of disability issues are also left for BoLSA as a double burden". The issues of disability run down by BoLSA as a paymaster. It is a double burden for BoLSA to possess the issue of PWDs in one hand and coordinating the whole labor and social issues among all sector bureaus on the other hand.

The manager of federation of PWDs explained the absence of responsible organizations on disability issues. He has the following to say:

If children abused, women are discriminated and youths stayed vacant, they have a responsible institution to hear their voice and find out solutions for problems, but who answers the quest of PWDs? No one, even BoLSA considers disability issues as a pity than a responsibility like other customary activities.

Participants indicated that the absence of specific governmental line ministry that dealt with specific responsibilities of disability equality resulted most disability issues ignored by officials and service providers. Because of such gaps, officials and practitioners give a deaf ear for the problems faced by PWDs. They failed to feel sense of belongingness on disability issues.

Similarly, discussants of the research also asserted the absence of specialist disability institutional structures. The absence of such institutional structures at the national level resulted mainstreaming led to the shutting down of dedicated disability units with the argument that all staff members are responsible for disability in the study sectors. One discussant explained, "Everybody’s activity is often no body`s responsibility." Because of absence of robust institutional structure and staff members with specific responsibilities of disability, disability issues failed to push into agenda. Part of the problem is a lack of political will to have a department that specifically handled disability affairs.

\section{Lack of involvement of PWDs and DPOs in planning and decision-making}

Dearth of involvement in planning and decision-making activities of disabled people and their organizations is 
another challenge for mainstreaming disability in the study sectors. This shows, often, people with disabilities excluded from decision-making in matters directly affecting their lives. The data from both interviews and FGDs confirmed likely on lack of involvement of PWDs and their organizations in planning and decision making as a challenge for mainstreaming disability into plans, programs and strategies of development. Regarding this, the Manager of federation of PWDs has the following affirmations to say:

It is unusual to observe PWDs in planning and decision making position from Kebele to higher authorities. Sometimes I feel that decisions are made by able-bodied than able-minded people. Even though we are disabled this is not to mean that we are in state of being unable to make things which requires mental ability rather it is to mean mental ability. You can take examples like what Merha Tsdeke ( a blind lawyer) and Yitenberesh Nigusie ( a blind lawyer, poet and social worker ) did for PWDs as a model which PWDs can do everything just like their non disabled peers if they get the chance to involve in planning and decision making activities.

Though PWDs have equal abilities in planning activities and making decisions they are ignored from involvements during action. In the study sectors, PWDs are not in positions, which plans developed and decisions are made. The plans and decisions that concerns PWDs made by other planners and decision makers in the essence of we know what PWDs want. As a result, in the plans developed and decisions set the heartfelt needs of PWDs might become ignored which in turn shades the effort of disability equality and inclusive environment.

\begin{abstract}
Absence of budgeting
Absence of sufficient funding to run the program of disability mainstreaming found out as a barrier that trudged the practice of disability mainstreaming in the study sectors. The data obtained through interviews conducted with office heads and focal persons including with planners and experts who involved in the FGDs show there is no budget specifically allocated for disability in general and disability-mainstreaming program in particular.

The absence of funds to run the issue of disability on different areas of practice affects the availability of quality services and made disastrous to reach all targeted beneficiaries. Absence of budgeting for disability exposed the study sectors to knock the door of other organizations for assistance, failed to equip with necessary office materials and created difficulties for celebrating disability day that is imperative for community mobilization. Economics of disability needed for direct cost of treatment and rehabilitation. However, the absence of appropriate funding from the central government hampered the provision of services in the study sectors and even deterred the success of designed programs and exposed PWDs for other difficulties.
\end{abstract}

\title{
Institutional and team loafing
}

The data obtained from participants of the research indicated the presence of organizational and team loafing culture as a challenge that restrained the practice of disability mainstreaming among the selected sector bureaus of the study. It makes responsible organs from managers to staffs run away from taking duties for practicing disability mainstreaming.

The loafing culture of institutions and teams goes beyond the possessor organization and sector bureaus of the study. The data from interviews revealed lack of commitment makes officials in different sector bureaus reluctant on the issue of disability. The deputy manager of education office reflected that, "When BoLSA summons different officials for discussing on issues related with disability I don't remember the time that more than half of the attendants come and discuss on the issue for discussion but if the case is for children, youths or women the hall becomes crowded". The culture of institutional and team loafing also resulted negligence on staffs in practicing disability mainstreaming. An interview with disability focal person explained, "Staffs in our organization are not committed to take the issue of disability mainstreaming as their own responsibility rather they solely transformed the responsibility for the focal person and coordinating expert of the case team".

\section{Lack of awareness}

Another challenging issue for the practice of disability mainstreaming program in the study sectors were the presence of awareness gaps with officials and staffs towards the program of disability mainstreaming. Awareness problem of officials and staffs created misunderstanding on the concept of disability mainstreaming among practitioners of the program. The manager of federation of PWDs described the unfamiliarity of responsible persons and staffs with the issue of disability mainstreaming program.

\section{Lack of follow up and nominal power of the responsible organization}

Monitoring and evaluation is helpful to identify the success and failures of the program, which, in turn helps to keep the strengths and adjust appropriate strategies for weakness. Such systems are helpful to examine the extent to which the programs of line sectors are responsive to the needs of PWDs. In this assumption, system of monitoring and evaluation of disability mainstreaming scrutinized in this study. Regarding this, data obtained through interview and FGDs revealed insufficient follow up and nominal power of the responsible organizations 
challenged the practice of disability mainstreaming into every aspect of development issues.

\section{Poor documentation and reporting}

Although different activities done at synthesizing service providers and the wider community aimed on the rights of PWDs, the documentation and reporting of such activities are limited and unorganized in the study sectors. Data obtained from the participants of the research confirmed the presence of poor documentation and reporting mechanisms in the study sectors challenged the process of disability mainstreaming. Study participants asserted that activities performed on the area of disability are not documented and arbitrarily reported which in turn create difficulties in identifying what worked, what didn't and why. The gaps on developing appropriate system of documentation and reporting restrained organizations from identifying their success and failures. Such failures further discouraged sector bureaus culture of devising new strategies for their failures.

\section{Hopelessness and sentimentality of PWDs}

The challenges for mainstreaming disability program were not limited to institutional, budgetary and practitioners problems. PWDs themselves also challenged the effective practice of disability mainstreaming in the study sectors. PWDs are become passive victims that failed to resist for their rights. A disability focal person in TVET office explained, "When PWDs faced a challenge in the process of service delivery they simply seclude from receiving services". He further added, "They expect everything to be in the safe side for them which are ideal. Even they did not find another strategies to address their problems rather they bitterly dislike the organizational services and give up for further solutions". PWDs are not in the position to enforce service providers to respect their rights and provide services that they are eligible for rather they associate the disruption of services with their disabilities and become hopeless for struggling to access services.

\section{Conclusion}

There are visible and encouraging trends in the study sectors regarding mainstreaming disability into development plan, implementation, monitoring and evaluation process. The study sectors mainstreamed disability through designing appropriate programs, developing human capacity, creating linkages, assigning disability point person and developing manuals and checklists. It is true that aspiring for disability mainstreaming through devising such strategies of practice is one-step forward by itself. However, there are serious limitations in effective implementations of the aforementioned strategies of disability mainstreaming. Most of the designed special programs trapped on paper, capacity-building activities are limited in quality and coverage, amateurish, double burdened disability point persons appointed and developed manuals, and checklists remained only at the hand of managers and focal person of the study sectors. This shows, although various strategies employed by study sectors to mainstream disability into development, they are not comprehensively implemented yet.

The practice of disability mainstreaming program in the study areas experience serious interlocked challenges emanated from the government, organizations, service providers and service beneficiaries. The hindering factors of disability mainstreaming in the study sectors are associated with absence of robust institutional structure, lack of involvement of PWDs and OPDs in planning and decision-making process and absence of budgeting. The practice of mainstreaming disability into development also affected by institutional and team loafing, hopelessness and sentimentality of PWDs, lack of awareness, dearth of follow up and nominal power of responsible organizations and poor documentation and reporting system. The government miscarried to launch disability responsible ministry and allocate appropriate budget for disability issues. Disability often left out and poorly understood in the context of development, PWDs and DPOs lacked voice at different level of society and attempts to identify and meet their needs have rarely occurred in the study areas. Organizations and staffs loosely follow and take measures on disability concerns and negligent to develop appropriate system of documentation and reporting. These impeding factors of disability mainstreaming implied the existence of pejorative attitudes towards disability issues at different levels of the study sectors. On the other hand, hopeless and seclude character of service users on the process of service provisions challenged the practice of disability mainstreaming program. All these implied, factors that deterred the practice of disability mainstreaming program in the study sectors are two-sided. They emanated from both organizational and personal problems.

\section{Recommendation}

- Awareness creation activities for both service providers and users towards the concept of disability mainstreaming program, their roles in the process of the program and the rights of PWDs in the process of service delivery should be made.

- Newfangled and all-inclusive trainings for managers, disability focal persons, staffs and service users to facilitate opportunities for disability equality should be given by the concerned stakeholders.

- Joint action of governmental, non-governmental and civic associations towards PWDs has to strengthen through devising different strategies. 
- Advocacy efforts should be made for launching of new and specific disability responsible organizational structure

- advocate for the rights of PWDs and DPOs to involve in plans and decisions which concerns their interests should be done

\section{References}

Albert, B., Dube, A., \& Hansen, T. (2005). Has disability been mainstreamed in to development cooperation.

Bruijn, p. (2013). Lessons learned on inclusion of peoples with disability in the ICCO Gelibandha food security project for ultra poor women. Netherlands: ICCO.

Bureau of Labor \& Social Affairs. (2015). Disability mainstreaming manual. Bahir Dar : BOLSA.

Central Statistical Agency. (2007). Population and housing census. Addis Abeba: CSA.

Dublin. (2004). Combat poverty agency, the forum of people with disabilities, national rehabilitation board, disability, exclusion \& poverty. London: Disability Alliance

Federal Democratic Republic of Ethiopia. (1995). The constitution of the Federal Democratic Republic of Ethiopia. Ethiopia: Addis Ababa.

HANDICAP international (2009). Let's do it! Mainstreaming disability into development policies, sector plans and practice. Cambodia: HANDICAP international.

HANDICAP international. (2013). Mainstreaming disability into disaster risk reduction: a training manual. Nepal: HANDICAP international.

International Labor Organization. (2013). Inclusion of people with disabilities in Ethiopia. ILO: Irish aid.

Jones, D. \& Webster, L. (2006). A handbook on disability mainstreaming. Indonesia: Solid Color Ltd.

Kassahun y. (2013). Law and policy situation of persons with disabilities in Ethiopia. Presentation given at the disability and risk management conference. Addis Ababa, October.

Light for the World. (2012). Strong together: policy plan (2011-2015). Retrieved from: http://www.un.org/disabilities.

Mindahun G. (2006). Employment opportunities of and discrimination against peoples with disability: the case of blind and physically impaired. Addis Ababa University, School of Graduate Studies, School of Social Work, M.A. thesis, Addis Ababa.

Ministry of Finance and Economic Development. (2010). The federal democratic republic of Ethiopia. Growth and transformation plan (GTP) (2010/11-14/15. Addis Abeba:MoFED

Ministry of Labor and Social Affairs. (2009). National employment policy and strategy of Ethiopia. Addis Abeba: MOLSA.

Mohapatra, S. (2012). In the absence of comparable information, most of the policy planning for people with disabilities is based on assumptions. Disability News and Information services, 9 (4). Retrieved from: http://www.dnis.org/interview.php?issue-id $=4 \&$ volume-id $=9 \&$ intervie-id $=190$.

Tana Forum. (2015). Tana high-level forum on security in Africa. Retrieved from: http://www.tanaforum.org

The Economist Intelligence Unit. (2014). Moving from the margins: Mainstreaming persons with disabilities in Pakistan. Retrieved from: http:// www.eiu.com

United Nations. (2006). The millennium development goals report. New York: UN

United Nations. (2011). Best practices for including persons with disabilities in all aspects of development efforts. $\mathrm{UN}$

Yeo, R. (2005). Disability, poverty and the new development agenda: Disability knowledge and research. UK: Manchester. 